\title{
Acacia mangium tannin as formaldehyde scavenger for low molecular weight phenol- formaldehyde resin in bonding tropical plywood
}

\begin{abstract}
One of the limitations in using low molecular weight phenol-formaldehyde (LmwPF) resin as a binder for wood-based panels is the amount of the free formaldehyde being emitted during soaking, pressing and sometimes during the earlier stage of application. Tannin from bark extracts is rich in phenolic compounds, and thus may be able to absorb this free formaldehyde and at the same time provide strength to the joint. In this study, tannin-phenol-formaldehyde adhesives were prepared by blending Acacia mangium bark extracts ( $40 \%$ solids) with low molecular weight phenol-formaldehyde ( $40 \%$ solids at 1:1 ratio). The tannin-LmwPF adhesive produced cured within 4 min at $130^{\circ} \mathrm{C}$, reduced the free formaldehyde to level $\mathrm{E} 1$ of European norm EN-120. The 3-ply plywood had acceptable shear strength (>1.0 MPa) exceeding the minimum requirements of European norms EN-314-1 and EN-314-2:1993 for interior and exterior applications, respectively. The study has shown that Acacia mangium tannin can be used as formaldehyde scavenger in LmwPF resin without compromising the strength of the joints.
\end{abstract}

Keyword: Formaldehyde scavanger; Tannin; Acacia mangium; Plywood; Low molecular weight phenol-formaldehyde 